\title{
Serum pyruvate dehydrogenase kinase as a prognostic marker for cholangiocarcinoma
}

\author{
SURANGKANA SANMAI $^{1}$, TANAKORN PROUNGVITAYA ${ }^{1}$, TEMDUANG LIMPAIBOON ${ }^{1,2}$, \\ DARAPORN CHUA-ON ${ }^{1}$, WUNCHANA SEUBWAI ${ }^{3}$, SITTIRUK ROYTRAKUL ${ }^{4}$, \\ SOPIT WONGKHAM ${ }^{2,5}$, CHAISIRI WONGKHAM ${ }^{2,5}$, ONGART SOMINTARA ${ }^{6}$, \\ SAKKARN SANGKHAMANON $^{7}$ and SIRIPORN PROUNGVITAYA ${ }^{1,2}$ \\ ${ }^{1}$ Centre for Research and Development of Medical Diagnostic Laboratories, \\ Faculty of Associated Medical Sciences; ${ }^{2}$ Cholangiocarcinoma Research Institute; \\ ${ }^{3}$ Department of Forensic Medicine, Faculty of Medicine, Khon Kaen University, Khon Kaen 40002; \\ ${ }^{4}$ National Center for Genetic Engineering and Biotechnology, National Science and \\ Technology Development Agency, Pathumthani 12120; Departments of ${ }^{5}$ Biochemistry, ${ }^{6}$ Surgery and \\ ${ }^{7}$ Pathology, Faculty of Medicine, Khon Kaen University, Khon Kaen 40002, Thailand
}

Received September 14, 2018; Accepted February 11, 2019

DOI: $10.3892 / \mathrm{ol} .2019 .10185$

\begin{abstract}
Pyruvate dehydrogenase kinase (PDK) is a Ser/Thr kinase that inactivates mitochondrial pyruvate dehydrogenase and serves a key role in aerobic glycolysis, which is a hallmark of cancer cells. The present study determined the PDK expression in cholangiocarcinoma (CCA) tissues and sera to evaluate their applicability as a biomarker for CCA. Using proteomic analysis, PDK was revealed to be the most overexpressed mitochondrial protein in CCA tissues. Then, the expression of PDK isoforms in CCA tissues was examined in 15 CCA cases by immunohistochemistry. The PDK3 isoform levels in the sera were measured using a dot blot assay for 39 patients with CCA, 20 patients with benign biliary disease and 19 healthy volunteers. The results revealed a 27-fold overexpression of PDK 3 in cancerous tissues when compared with adjacent non-cancerous tissues. The immunohistochemical results demonstrated that the PDK1, 2 and 3, but not the PDK4, isoforms were overexpressed in cancerous tissues. When the PDK3 levels in the sera were examined, they were significantly higher in CCA when compared with the BBD and healthy groups. The specificity and sensitivity of PDK3 as a marker for CCA were 97.5 and $33.0 \%$, respectively, and high PDK3 levels in the sera were correlated with a short survival time for CCA.
\end{abstract}

Correspondence to: Dr Siriporn Proungvitaya, Centre for Research and Development of Medical Diagnostic Laboratories, Faculty of Associated Medical Sciences, Khon Kaen University, 123 Moo 16 Mittapap Road, Nai-Muang, Muang, Khon Kaen 40002, Thailand

E-mail: sirpat@kku.ac.th

Key words: pyruvate dehydrogenase kinase, cholangiocarcinoma, serum, prognostic marker
In conclusion, PDK3 can be used as a diagnostic/prognostic marker for CCA.

\section{Introduction}

Cholangiocarcinoma (CCA) is a tumor originating from biliary epithelial cells. The incidence of CCA in Southeast Asia, especially in northeast Thailand, is remarkably high with liver fluke infection as a major risk factor. The prognosis of CCA is poor with high mortality rates as the majority of patients are diagnosed at a late stage with a high incidence of metastasis (1-3). Reliable diagnostic/prognostic markers are critical for CCA treatment. For CCA diagnosis, there are several markers which are reported to be effective diagnostic and prognostic markers such as carcinoembryonic antigen, carbohydrate antigen (CA) 19-9, CA242, CCA-associated carbohydrate antigen, mucin glycoproteins and cytokines (4). Even combinations of those markers are still insufficient for sensitivity and specificity (5).

Recently, using mass spectrometry, Chua-On et al (6) demonstrated the different expressions of mitochondrial proteins in cancerous and adjacent tissues of CCA. Since the main energy of cancer cells is produced by glucose metabolism, the glucose metabolism involved-proteins in the mitochondria could be potential diagnostic/prognostic markers. Among all the candidate proteins, pyruvate dehydrogenase kinase (PDK) 3 showed the highest fold increase. PDK is a Ser/Thr kinase that inactivates mitochondrial pyruvate dehydrogenase by site-specific phosphorylation and plays a key role in regulation of the Warburg effect in cancer cells (7). There are four isoforms, including PDK1, PDK2, PDK3 and PDK4. Their molecular weight range from $45 \mathrm{kDa}$ (PDK1) to $48 \mathrm{kDa}$ (PDK2, PDK3 and PDK4) and models show that they have $70 \%$ identity of themselves (8). All PDK isoforms are notably expressed in specific tissues (9). At present, there 
is no report of PDK expression in CCA. The aims of this study were to examine PDK expression in CCA and evaluate whether the PDKs could be diagnostic/prognostic markers of CCA.

\section{Materials and methods}

Selection of PDK from the mitochondrial protein database. Glucose metabolism-associated proteins were selected from the CCA mitochondrial proteomic database constructed by Chua-On et al (6), of which data were extracted from three CCA tissues and adjacent non-cancerous tissues using liquid chromatography tandem-mass spectrometry. Proteins were searched using Mascot against The National Center for Biotechnology Information human protein reference database with the human mitochondrial proteins database. The 282 proteins overexpressed in CCA were classified into their respective biological functions using Protein Analysis Through Evolutionary Relationships (PANTHER) software (10).

CCA tissues and sera. Cancerous and adjacent non-cancerous tissues from 15 patients with intrahepatic CCA were kindly provided by the Cholangiocarcinoma Research Institute (CARI), Khon Kaen University, Khon Kaen, Thailand. They were immediately snap-frozen in liquid nitrogen and stored at $-80^{\circ} \mathrm{C}$ until further use. A part of each frozen tissue section was fixed in $4 \%$ buffered formalin and processed for hematoxylin and eosin staining for histopathological diagnosis. The mean \pm standard deviation (SD) of age, aspartate transaminase, alanine transaminase and alkaline phosphatase (ALP) of these patients are $57 \pm 5.5$ years, $61 \pm 6.7,57 \pm 51.4$ and $154 \pm 100.4 \mathrm{U} / 1$, respectively.

Serum samples from 39 patients with CCA and 20 patients with benign biliary diseases (BBD), mostly cholangitis, were additionally obtained from The Faculty of Medicine, CARI, Khon Kaen University. Normal control sera were obtained from 19 healthy people, who went for check-ups at the Office for Medical Technology and Physical Therapy Health Service, Faculty of Associated Medical Sciences, Khon Kaen University. Characteristics of the healthy controls, and patients with BBD and CCA are summarized in Table I. The serum samples were kept at $-20^{\circ} \mathrm{C}$ until further use.

The sample sizes for CCA tissues and serum samples were analyzed using PS program version 3.1.2 (11). This research project was approved by the Ethical Committee of Khon Kaen University, Thailand (approval no. HE581431).

Immunohistochemistry. Paraffin-embedded CCA sections of $4 \mu \mathrm{m}$ thickness of 15 CCA cases were deparaffinized by soaking in xylene, absolute ethanol, $95 \%$ ethanol and $70 \%$ ethanol for 2 min each time. For the antigen retrieval process, sections were boiled in $1 \mathrm{X}$ citrate buffer ( $\mathrm{pH}$ 6.0) for $10 \mathrm{~min}$ and washed in $1 \mathrm{X}$ PBS buffer. Then, the endogenous peroxidase activity of the sections was blocked with $3 \% \mathrm{H}_{2} \mathrm{O}_{2}$ in methanol for $1 \mathrm{~h}$ in the dark and non-specific background binding was blocked by incubation with $20 \%$ fetal bovine serum (Sigma-Aldrich; Merck KGaA, Darmstadt, Germany ) for $2 \mathrm{~h}$. The sections were then incubated with $150 \mu \mathrm{l}$ rabbit polyclonal antibody against human PDK1, PDK2, PDK3 and
PDK4 (Biorbyt, Cambridge, UK; cat. nos. of the anti-PDK1, -PDK2, -PDK3 and -PDK4 antibodies are orb14422, orb137873, orb312719 and orb136059, respectively) at a dilution of $1: 100$ at $4^{\circ} \mathrm{C}$ overnight. The sections were washed in 1X PBS-T and incubated with $150 \mu 1$ undiluted goat anti-rabbit immunoglobulin $\mathrm{G}(\mathrm{IgG})$ antibody with the EnVision System (Dako; Agilent Technologies, Inc., Santa Clara, CA, USA) for $1 \mathrm{~h}$ and the signal was developed with diamino-benzidine (Dako; Agilent Technologies, Inc.) for $5 \mathrm{~min}$ in the dark. The sections were washed with running tap water until clear and counterstained with hematoxylin for $10 \mathrm{~min}$. The sections were dehydrated in an ascending series of ethanol for $2 \mathrm{~min}$ at each concentration, cleared in xylene, mounted with permount and sealed with a cover glass.

To assess the immunohistochemical staining (IHC) using $\mathrm{H}$-score, both the intensity of staining ( 0 , no staining; $1+$, weak staining; $2+$, moderate staining; and $3+$, strong staining) and the percentage of the stained tumor cells $(0-100 \%)$ were assessed. IHC results were observed for $\sim 10$ fields per sample to reduce variation of detection. The $\mathrm{H}$-score was calculated as a sum of the intensity as follows (12): $\mathrm{H}$-score $=(\%$ of positively stained tumor cells at weak intensity $x 1)+(\%$ of positively stained tumor cells at moderate intensity $\mathrm{x} 2)+(\%$ of positively stained tumor cells at strong intensity x3). Lastly, 10 fields of $\mathrm{H}$-score were averaged leading to a range of 0-300 for the $\mathrm{H}$-score for each sample.

Western blot analysis. In total, five CCA sera samples ( $2 \mu 1$ each) were mixed with $4 \mathrm{X}$ loading dye to a 1:4 ratio and boiled for $5 \mathrm{~min}$. The protein samples were fractionated on $12.5 \%$ SDS-PAGE and run at $120 \mathrm{~V}$ for $3 \mathrm{~h}$ in a cold room. The electrophoresis was completed when the dye reached the end of the gel, and the separated proteins were transferred onto a polyvinylidene difluoride membrane at $300 \mathrm{~V}$ for $1 \mathrm{~h}$ at room temperature. The membrane was blocked with $5 \%$ skimmed milk in 1X TBS with Tween-20 (TBST) for $1 \mathrm{~h}$ at room temperature. The membrane was then incubated with a 1:500 dilution of primary antibody, which was a rabbit polyclonal antibody against human PDK3 overnight at $4^{\circ} \mathrm{C}$. Next, the membrane was washed three times with $1 \mathrm{X}$ TBST for 10 min each time, and incubated with a 1:10,000 dilution of anti-rabbit $\mathrm{IgG}$-horseradish peroxidase-conjugated secondary antibody for $1 \mathrm{~h}$ at room temperature, followed by three washes with $1 \mathrm{X}$ TBST for $10 \mathrm{~min}$ each time. Finally, the chemiluminescence was detected using an Enhanced Chemiluminescent plus system (GE Healthcare Life Sciences, Little Chalfont, UK) and visualized using Image Quant LASmini 400 (GE Healthcare Life Sciences). A KKU-055 (a CCA cell line) lysate was used as a positive control for PDK3, which was provided by CARI, Khon Kaen University.

Dot blot assay. The membrane was soaked in 1X TBST for 10 min before being placed on a machine. All sera of each group ( $2 \mu 1$ each) were transferred to a nitrocellulose membrane with Bio-Dot Microfiltration Apparatus (Bio-Rad Laboratories, Inc., Hercules, CA, USA). After air-drying, the membrane was blocked with 5\% skimmed milk in $1 \mathrm{X}$ TBST for $1 \mathrm{~h}$ at room temperature. The membrane was then incubated with a 1:500 dilution of rabbit polyclonal antibody against human PDK3 
Table I. Characteristics of the healthy controls, and the benign biliary disease and cholangiocarcinoma patients.

\begin{tabular}{lccrr}
\hline Clinical parameters (normal range) & Healthy control $(\mathrm{n}=19)$ & BBD $(\mathrm{n}=20)$ & CCA $(\mathrm{n}=39)$ & P-value \\
\hline Age, years & $49.8 \pm 5.4(40,59)$ & $59.3 \pm 9.3(41,74)$ & $59.3 \pm 9.3(38,77)$ & $<0.0002^{\mathrm{b}, \mathrm{c}}$ \\
Sex, $\mathrm{n}(\%)$ & & & & \\
Male & $4(21.1)$ & $16(80.0)$ & $23(59.0)$ & $0.0009^{\mathrm{b}, \mathrm{c}}$ \\
Female & $15(78.9)$ & $4(20.0)$ & $16(41.0)$ & \\
Liver function enzymes & & & & \\
ALT (4-36 U/l) & $19.1 \pm 5.5(8,28)$ & $88.0 \pm 101.4^{\mathrm{a}}(8,429)$ & $57.5 \pm 43.0(2,151)$ & $<0.0001^{\mathrm{d}, \mathrm{e}}$ \\
AST (12-32 U/l) & $25.5 \pm 4.2(19,31)$ & $90.0 \pm 75.3^{\mathrm{a}}(16,303)$ & $84.8 \pm 76.3(19,317)$ & $<0.0001^{\mathrm{d}, \mathrm{e}}$ \\
ALP (42-121 U/l) & $73.6 \pm 16.0(45,105)$ & $332.8 \pm 285.7^{\mathrm{a}}(32,991)$ & $226.9 \pm 307.9(24,1963)$ & $<0.0001^{\mathrm{d}, \mathrm{e}}$ \\
\hline
\end{tabular}

Values are presented as the mean \pm standard deviation, (minimum, maximum). The characteristics of three independent sample groups were analyzed using the Kruskal-Wallis test. ${ }^{\mathrm{a}}$ The number of samples analyzed was 18 due to a limitation in the amount of patient data; ${ }^{\mathrm{b}} \mathrm{P}<0.001$, Healthy controls vs. BBD; ${ }^{\mathrm{P}}<0.001$, healthy controls vs. CCA; ${ }^{\mathrm{d}}<0.0001$, healthy controls vs. $\mathrm{BBD}$; ${ }^{\mathrm{e}} \mathrm{P}<0.0001$, healthy controls vs. CCA. ALT, alanine aminotransferase; AST, aspartate aminotransferase; ALP, alkaline phosphatase; BBD, benign biliary disease; CCA, cholangiocarcinoma.

(Biorbyt) overnight at $4^{\circ} \mathrm{C}$. The detection process was the same as the western blot analysis method. The method was modified from a previously described method (13). A HeLa cell lysate was used as a positive control. The cell lysate was kindly provided by Daraporn Chua-orn (Khon Kaen University). The intensities of PDK3 protein in the sera were normalized using PDK3 intensity in the HeLa cell lysate as a relative expression ratio.

Receiver operating characteristic (ROC) curve analysis. ROC curve analysis was performed to analyze the applicability of PDK3 as a tumor marker for CCA. The cut-off value was considered in the left-top or shoulder of the curve. In this study, we selected the value that provided the highest likelihood ratio as the cut-off.

Statistical analysis. Fold change analysis was calculated from the signal intensity of CCA minus the signal intensity of adjacent non-cancerous tissue. The data are presented as mean \pm standard deviation and the range (minimum to maximum). A Kruskal-Wallis normality test was used for the normality of data. The different values among two and three sample groups were estimated using the Mann-Whitney and Kruskal Wallis tests, respectively. Bonferroni's correction was used as the post hoc test. The correlation between serum PDK3 levels and the clinicopathological parameters of the patients were analyzed using Fisher's exact test. $\mathrm{P}<0.05$ was considered to indicate a statistically significant difference. GraphPad Prism v.5 software (GraphPad Software, Inc., La Jolla, CA, USA) was used for statistical analyses.

\section{Results}

PDK expression in CCA. In our previous study, the different expression of mitochondrial proteins in cancerous and adjacent normal tissues of CCA specimens was determined using mass spectrometry, and it was identified that a total of 281 mitochondrial proteins were identified to be overexpressed in CCA tissues (6). All 281 proteins were considered to be significantly different $(\mathrm{P}<0.05)$. In this study, we classified those 281 mitochondrial proteins into their respective biological function using PANTHER software (10) (analyzed on 21st August 2017). The results showed that the majority of the proteins were classified into cellular process $(82,29.1 \%)$, metabolic process $(80,28.4 \%)$, cellular component organization or biogenesis $(28,9.9 \%)$, localization $(23,8.3 \%)$ and other processes possibly associated with cancer, such as biogenesis and immune system (Fig. 1). Then, we focused on the proteins involved in metabolic process and identified 15 proteins that are involved in glucose metabolism. Among those 15 proteins, PDK3 was the most overexpressed protein. In addition, a 6-fold overexpression of PDK 2 was detected (Table II).

High expression of PDK1, PDK2 and PDK3 in CCA tissues. To evaluate the expression of four PDK isoforms in CCA, immunohistochemistry was performed on $15 \mathrm{CCA}$ tissues from patients containing cancerous and adjacent non-cancerous tissues. The results showed that PDK1, 2 and 3 were strongly positive in the cancer cells but only weakly positive in adjacent non-cancerous tissues. However, PDK4 was negative in both cancerous and non-cancerous adjacent tissues (Fig. 2A). The intensities of immunostaining of PDK1, PDK2 and PDK3, expressed by $\mathrm{H}$-scores (mean $\pm \mathrm{SD}$ ), were $269 \pm 34,268 \pm 32$ and $268 \pm 20$, respectively, in CCA tissues. Whereas, the H-scores of adjacent non-cancerous tissues were $38 \pm 28,30 \pm 21$ and $54 \pm 11$, respectively (P-value $<0.0001$; Mann-Whitney U test; Fig. 2B).

High expression of $P D K 3$ in the sera of patients with CCA. The levels of PDK1, PDK2 and PDK3 in the sera were measured in 39 patients with CCA, 20 patients with BBD and 19 normal controls using dot blot analysis. We confirmed that PDK3 expression in sera was not influenced by age and sex by statistical testing in all three groups (Tables SI and SII). 
Table II. Candidate mitochondrial proteins involved in the glucose metabolism of cholangiocarcinoma.

\begin{tabular}{llc}
\hline Protein name & Gene symbol & Fold change of CCA $^{\text {a }}$ \\
\hline Pyruvate dehydrogenase kinase 3 & PDK3 & 27 \\
Cytochrome $c$ oxidase assembly protein 3 homolog & COX15 & 11 \\
Cytochrome $c$ oxidase subunit 5A & COX5A & 10 \\
ATP synthase subunit epsilon & ATP5E & 11 \\
Cytochrome $c$ oxidase subunit 5b & COX5B & 7 \\
Complex I intermediate-associated protein 30 & CIA30 & 7 \\
Succinate dehydrogenase & SDH & 7 \\
Pyruvate dehydrogenase kinase 2 & PDK2 & 6 \\
Acetyl-CoA carboxylase 2 & ACC2 & 8 \\
NADH dehydrogenase [ubiquinone] 1 alpha subcomplex subunit 2 & NDUFA2 & 4 \\
ATP synthase subunit alpha, mitochondrial isoform & ATP5A & -1 \\
Cytochrome b-c1 complex subunit 1 & UQCRC1 & -3 \\
ATP synthase mitochondrial F1 complex assembly factor 1 & ATPAF1 & 4 \\
Isocitrate dehydrogenase & IDH & -4 \\
Cytochrome $c$ oxidase subunit I & COX1 & 0
\end{tabular}

${ }^{a}$ Fold change was calculated from the signal intensity from mass spectrometry of CCA minus the signal intensity of adjacent non-cancerous tissues. The signal intensity from mass spectrometry was presented as $\log 2$ values. Once the differences were calculated, the values were multiplied by 2. CCA, cholangiocarcinoma.

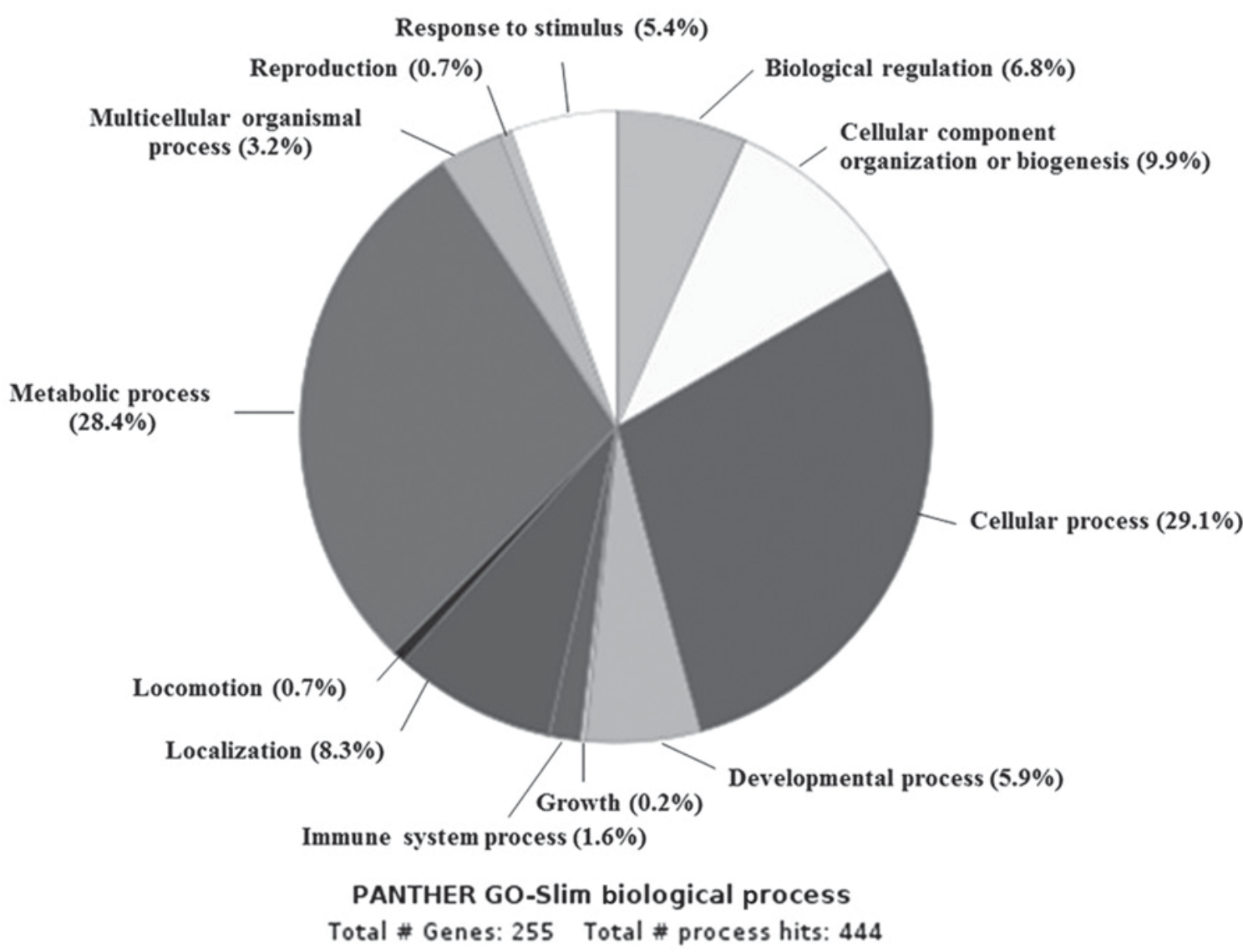

Figure 1. Mitochondrial protein classification according to protein class. GO, Gene Ontology.

As shown in Fig. 3, PDK3 was significantly higher in CCA sera compared with normal sera $(\mathrm{P}=0.03)$. There was no significant difference in PDK3 levels between BBD and normal sera. Furthermore, the comparison of the PDK3 
Table III. Correlation between low and high pyruvate dehydrogenase kinase 3 levels and the clinicopathological characteristics of patients with cholangiocarcinoma.

\begin{tabular}{|c|c|c|c|c|c|}
\hline \multirow[b]{2}{*}{ Clinical parameters } & \multirow[b]{2}{*}{$\mathrm{n}$} & \multicolumn{2}{|c|}{ Low PDK3 } & \multicolumn{2}{|c|}{ High PDK3 } \\
\hline & & r-value & P-value & r-value & P-value \\
\hline Age (years) & 39 & 0.03168 & 0.8976 & -0.0144 & 0.9521 \\
\hline \multicolumn{6}{|l|}{ Liver function enzymes } \\
\hline $\operatorname{ALT}(\mathrm{U} / \mathrm{l})$ & 39 & 0.1986 & 0.4150 & -0.2878 & 0.2185 \\
\hline AST (U/l) & 39 & 0.2405 & 0.3213 & 0.0239 & 0.9205 \\
\hline $\operatorname{ALP}(\mathrm{U} / \mathrm{l})$ & 39 & 0.2368 & 0.3441 & -0.4837 & $0.0307^{\mathrm{a}}$ \\
\hline Total bilirubin (mg/dl) & 39 & 0.5035 & $0.0280^{\mathrm{a}}$ & -0.0140 & 0.9532 \\
\hline Direct bilirubin $(\mathrm{mg} / \mathrm{dl})$ & 39 & 0.4622 & $0.0463^{\mathrm{a}}$ & -0.0350 & 0.8836 \\
\hline \multicolumn{6}{|l|}{ Tumor markers } \\
\hline CEA (ng/ml) & 26 & 0.3571 & 0.2310 & -0.0572 & 0.8527 \\
\hline CA 19-9 (U/ml) & 32 & -0.1096 & 0.6861 & 0.1900 & 0.4810 \\
\hline Survival time (days) & 32 & 0.0161 & 0.9529 & -0.5385 & $0.0314^{\mathrm{a}}$ \\
\hline
\end{tabular}

${ }^{\mathrm{a}}<0.05$. PDK, pyruvate dehydrogenase kinase; ALT, alanine aminotransferase; ALP, alkaline phosphatase; AST, aspartate aminotransferase; CEA, carcinoembryonic antigen; CA, cancer antigen.

A

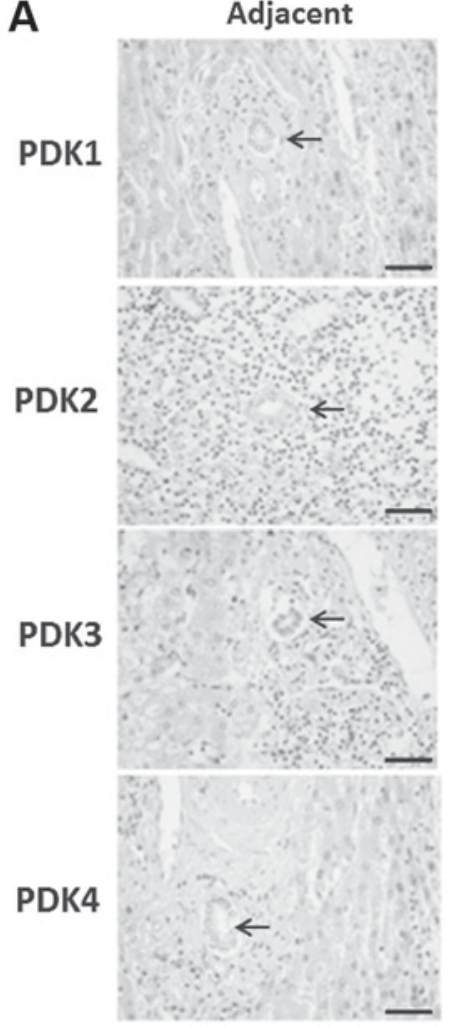

CCA

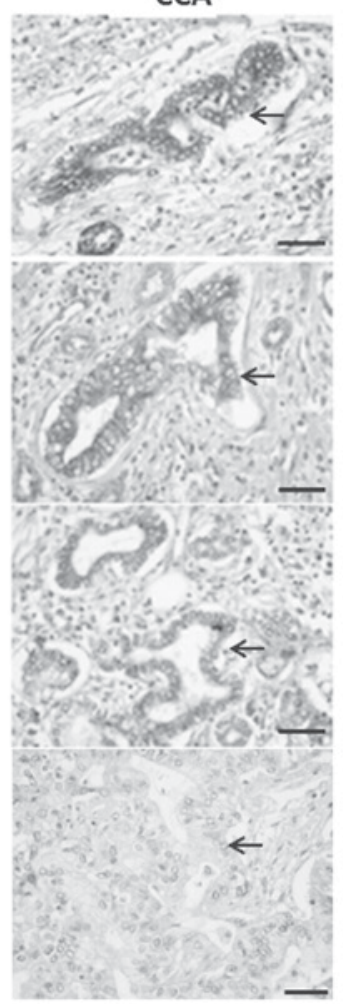

B

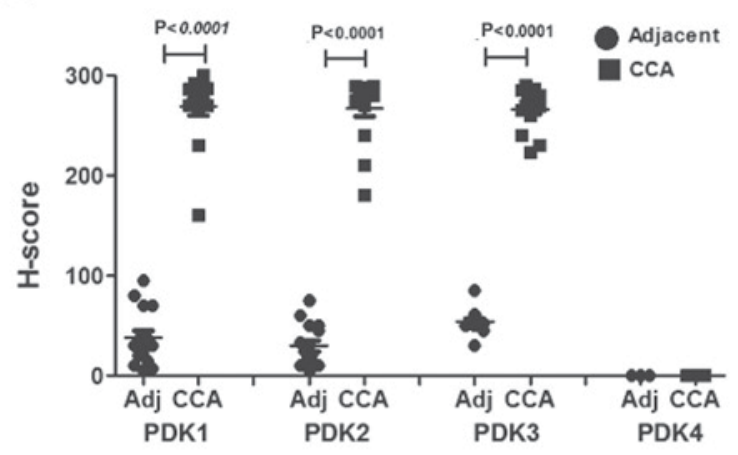

Figure 2. (A) Representative PDK staining in cancerous and non-cancerous tissues of the surgical specimens from patients with CCA (magnification, $\mathrm{x} 400$; scale bar, $50 \mu \mathrm{m}$ ). Arrows in the 'Adjacent' column indicate cholangiocytes, and arrows in the 'CCA' column indicate CCA cells. (B) PDK1, PDK2 and PDK3, but not PDK4, were significantly overexpressed in CCA tissues compared with adjacent non-cancerous tissues (P<0.0001). PDK, pyruvate dehydrogenase kinase; CCA, cholangiocarcinoma.

levels in the sera revealed that it was significantly higher in CCA sera compared with the BBD group sera at a P-value of 0.03 . For PDK1 and PDK2, the result showed no significant difference between CCA vs. normal control and CCA vs. BBD. To confirm that the dot blot positivity was in fact detecting PDK3, we performed western blot analysis for five representative dot-blot positive CCA sera. PDK3 was detected in all five CCA sera as a $48 \mathrm{kDa}$ band. The cell lysate of the 

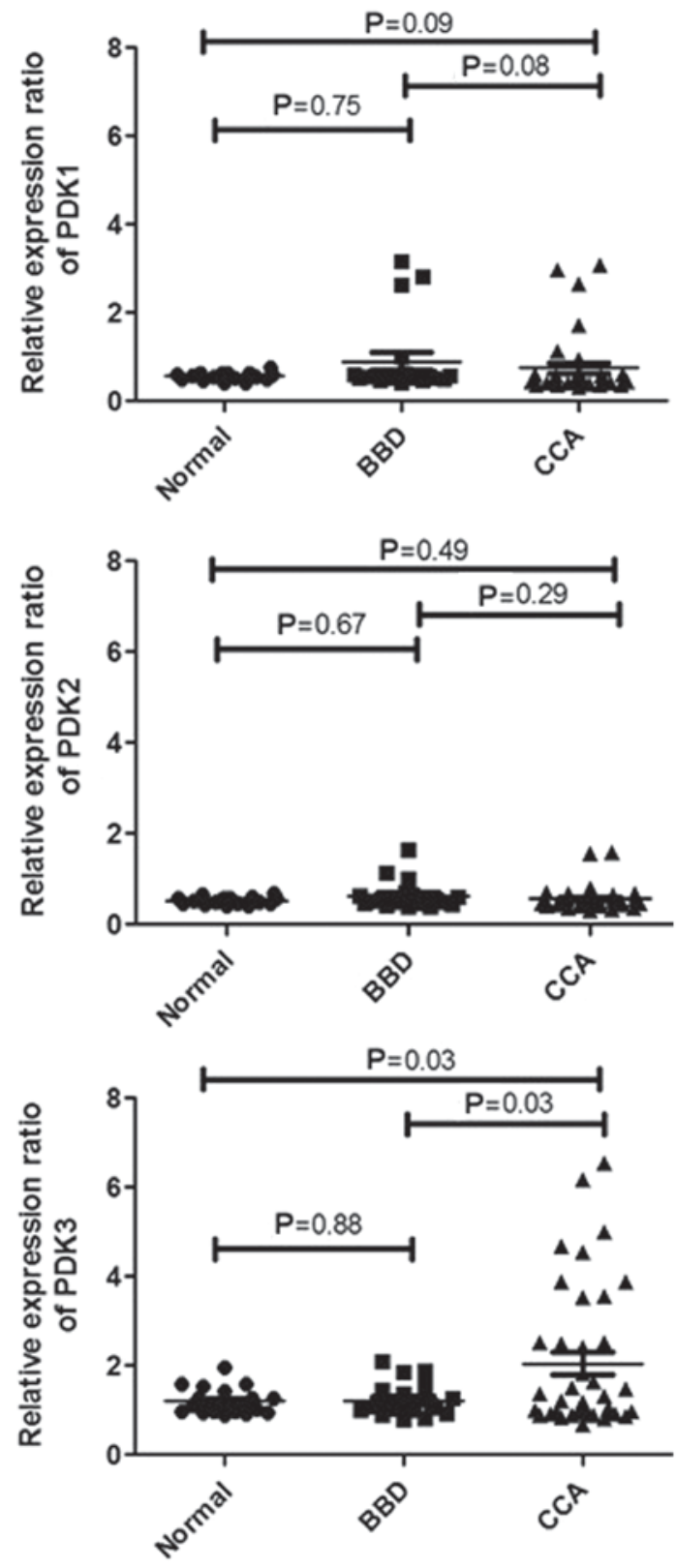

Figure 3. PDK1, PDK2 and PDK3 levels in the sera of CCA, BBD and healthy control groups. While PDK1 and PDK2 were rarely detected in all three groups, the PDK3 level in the CCA group was significantly higher when compared with the other two groups. Slightly less than a half of the patients with CCA exhibited high levels of PDK3 in the sera. PDK, pyruvate dehydrogenase kinase; $\mathrm{CCA}$, cholangiocarcinoma; $\mathrm{BBD}$, benign biliary disease.

KKU-055 CCA cell line was used as a positive control for PDK3 (Fig. S1).

PDK3 as a CCA tumor marker. The cut-off of PDK3 was calculated from an ROC curve and the value that provided the highest likelihood ratio was used as the cut-off. The PDK3 cut-off of CCA from non-CCA was 2.253 (Fig. 4). We found $33.0 \%$ sensitivity, $97.5 \%$ specificity, $92.9 \%$ positive predictive value, $60.0 \%$ negative predictive value and $65.8 \%$ accuracy.

Correlation of high PDK3 and short survival. We investigated the correlation between serum PDK3 levels with

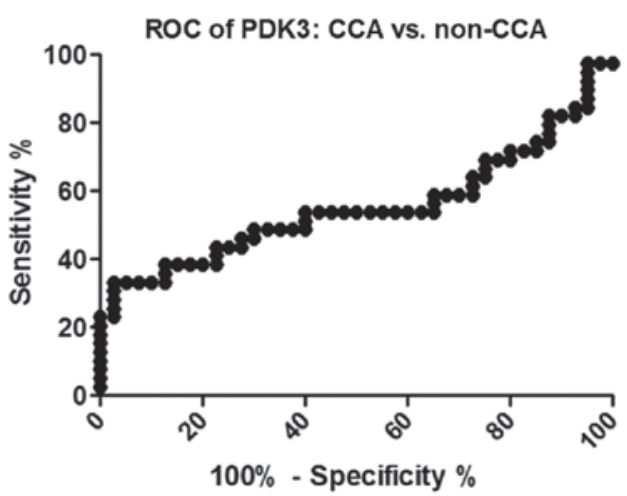

Figure 4. ROC curve of the PDK3 levels in CCA against non-CCA. The cut-off value of PDK 3 was calculated from the ROC curve. The cut-off of PDK3 to distinguish CCA from non-CCA was 2.253. ROC, Receiver Operating Characteristic; PDK, pyruvate dehydrogenase kinase; CCA, cholangiocarcinoma.

clinicopathological parameters of patients with CCA (Table III). Using the median cut-off, we found a negative correlation between the serum PDK3 level and the ALP level at a P-value of 0.0307 (Fig. 5A). Moreover, the serum PDK3 level was negatively correlated with the survival time of patients, as shown in Fig. 5B with a P-value of 0.0314 (Table III). This negative correlation indicated that PDK3 could be a prognostic marker for patients with CCA; the higher the PDK3 level, the shorter the survival time.

\section{Discussion}

Proteomic analysis on the expression of mitochondrial proteins revealed that the proteins involved in glucose metabolism are overexpressed in CCA. In particular, PDK3 showed the highest (27-fold) change in $>15$ proteins. By immunohistochemistry, the overexpression of PDK1, PDK2 and PDK3 was identified in CCA tissues compared with the adjacent non-cancerous tissues. From the existing literature, PDK1 and PDK2 are hypothesized to be strongly expressed in cancer cells because PDK1 is mainly associated with aerobic glycolysis, which is the preferred state for cancer cells to produce adenosine triphosphate, even in sufficient oxygen support $(14,15)$. For PDK2, there is a study that demonstrated PDK2 expression in the majority of normal tissues (9). We studied the expression of all PDK isoforms and found that PDK1, PDK2 and PDK3, but not PDK4, were significantly overexpressed in 15 CCA tissues. PDK1 overexpression was reported in gastric, renal and colon cancers (16-18). PDK2 was highly expressed in head and neck cancers (19). The high expression of PDK3 was reported in colon cancer (18). PDK3 inhibits pyruvate dehydrogenase activity via phosphorylation of the E1 subunit of pyruvate dehydrogenase E1 component subunit $\alpha$, somatic form, mitochondrial, and thereby regulates glucose metabolism and aerobic glycolysis in cancer. Moreover, there are previous studies that demonstrated that PDK3 is involved in anticancer resistance $(20,21)$.

We also studied the serum PDK levels in normal controls, and patients with BBD and CCA. Until now, the PDK level in the serum or plasma has not been determined in healthy controls and patients with diseases. As PDK is a 

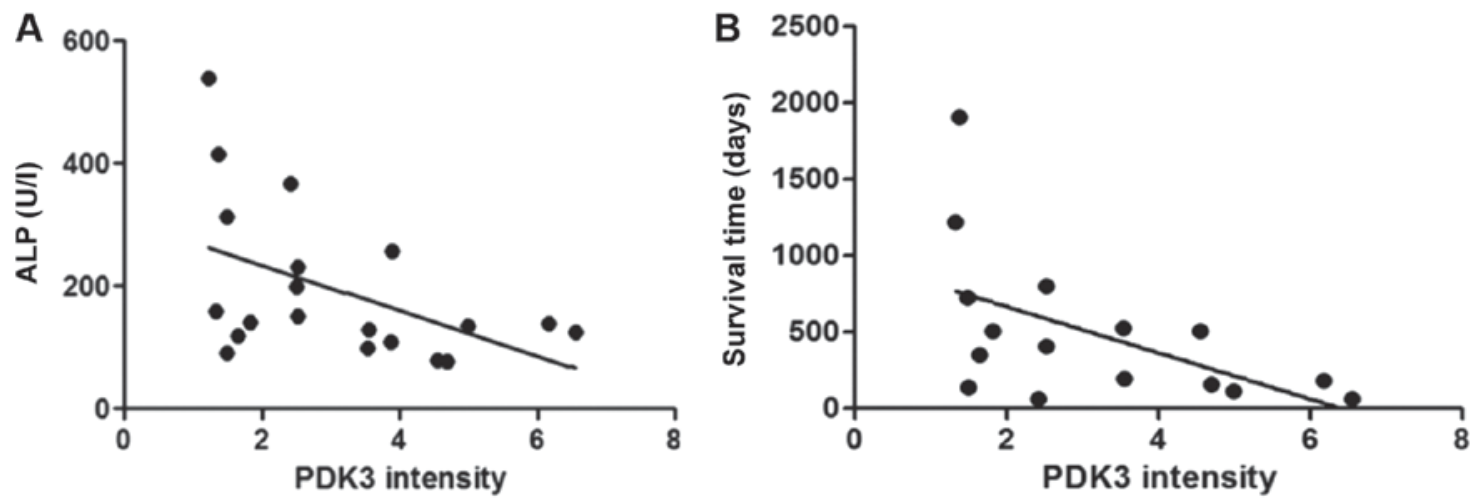

Figure 5. Significant correlation of PDK3 intensity with (A) ALP and (B) survival time. Inverse correlations were identified in PDK3 with ALP and survival time. PDK, pyruvate dehydrogenase kinase; ALP, alkaline phosphatase.

mitochondrial protein, it was surprising that PDK1, 2 and 3 were detected in the sera, especially in the sera of patients with CCA. When the secretion abilities of the PDKs were determined using SignalP 4.1 server with a cut-off D-score of 0.45 , the D-score of the PDKs was 0.126 , which suggested that PDKs are not secreted proteins (22). As SignalP is a server, which detects signal peptides of transmembrane regions, PDKs may be secreted from CCA through other pathways (22). While all CCA tissues were positive for PDK1, 2 and 3 by immunohistochemistry, only less than a half of CCA sera were positive for PDK3. This suggested that PDK3 was not always secreted from CCA for circulation and this secreted PDK3 has significance in some clinical aspects. Since the high serum PDK3 level is associated with the poor prognosis of the patients, the role of PDK3 in tumor invasion/metastasis as well as tumor proliferation requires examination in the future.

Although PDK3 provided good specificity to CCA in the IHC analysis of tumor tissues, its serum level presented a rather low sensitivity for discrimination of CCA and non-CCA. This discrepancy is due to the low detection rate of PDK3 in the sera of patients with CCA. In this aspect, PDK3 is not a good diagnostic marker. Nevertheless, when the correlation of the PDK3 intensity and clinical parameters of patients was analyzed, a significant correlation was observed between PDK3 and three CCA biomarkers, namely, ALP, total bilirubin and direct bilirubin. Our previous studies revealed that, in CCA, the total serum bile acid correlates with total bilirubin, and total serum bile acid and intercellular adhesion molecule 1 correlate with $\operatorname{ALP}(23,24)$. Although the possible mechanisms underlying this phenomenon remain unclear, an inverse correlation between the PDK3 level and survival times was observed. Therefore, the destructive nature of CCA may be associated with PDK3 release for circulation. The PDK3 level in serum could be an effective prognostic marker of CCA. However, limitation of this study was the small sample size so age- and sex-matched groups were unable to be gathered for CCA, BBD patients and normal controls.

In our study, PDK3 levels in the sera of CCA patients were significantly higher than both normal and BBD. Although serum PDK3 level was high in less than half of the CCA patients, high PDK3 level was significantly correlated with poor prognosis. These results indicated that PDK3 might be used as a prognostic marker of CCA.

\section{Acknowledgements}

The authors would like to thank Professor Yukifumi Nawa (Tropical Diseases Research Centre, Faculty of Medicine, Khon Kaen University, Khon Kaen, Thailand), for editing the manuscript.

\section{Funding}

The project was supported by grants from Khon Kean University (Khon Kaen, Thailand; grant no. 601804), the Centre for Research and Development of Medical Diagnostic Laboratories, Faculty of Associated Medical Sciences and the Cholangiocarcinoma Research Institute, Khon Kaen University, the Research Fund for Supporting Lecturer to Admit High Potential Student to Study and Research on His Expert Program Year 2014, Graduate School, Khon Kaen University (grant no. 571H107) and the Publication Clinic of the Research Affairs, Khon Kaen University.

\section{Availability of data and materials}

The datasets used and analyzed during this study are available from the corresponding author on reasonable request.

\section{Authors' contributions}

SSa performed the experiments and analyzed the data. TP, TL, WS and SP revised and conceived the study. SR developed the mass spectrometry database. DCO contributed to the CCA mitochondrial data analysis. SW and CW contributed to the study design and were involved in drafting the manuscript. OS and SSu collected the CCA tissue samples. All authors have read and approved the final version of the manuscript.

\section{Ethics approval and consent to participate}

This research project was approved by the Ethical Committee of Khon Kaen University, Thailand (no. HE581431). Written 
inform consent was obtained from all patients at the time of sample collection by the Cholangiocarcinoma Research Institute.

\section{Patient consent for publication}

Not applicable.

\section{Competing interests}

The authors declare that they have no competing interests.

\section{References}

1. Khan SA, Toledano MB and Taylor-Robinson SD: Epidemiology, risk factors, and pathogenesis of cholangiocarcinoma. HPB (Oxford) 10: 77-82, 2008.

2. Sripa B, Brindley PJ, Mulvenna J, Laha T, Smout MJ, Mairiang E, Bethony JM and Loukas A: The tumorigenic liver fluke Opisthorchis viverrini-multiple pathways to cancer. Trends Parasitol 28: 395-407, 2012.

3. Rizvi S and Gores GJ: Pathogenesis, diagnosis, and management of cholangiocarcinoma. Gastroenterology 145: 1215-1229, 2013.

4. Pattanapairoj S, Silsirivanit A, Muisuk K, Seubwai W, Cha'on U, Vaeteewoottacharn K, Sawanyawisuth K, Chetchotsak D and Wongkham S: Improve discrimination power of serum markers for diagnosis of cholangiocarcinoma using data mining-based approach. Clin Biochem 48: 668-673, 2015.

5. Wongkham S and Silsirivanit A: State of serum markers for detection of cholangiocarcinoma. Asian Pac J Cancer Prev 13 (Suppl): S17-S27, 2012.

6. Chua-On D, Proungvitaya T, Techasen A, Limpaiboon T, Roytrakul S, Wongkham S, Wongkham C, Somintara O, Sungkhamanon S and Proungvitaya S: High expression of apoptosis-inducing factor, mitochondrion-associated 3 (AIFM3) in human cholangiocarcinoma. Tumour Biol 37: 13659-13667, 2016.

7. Icard $\mathrm{P}$ and Lincet $\mathrm{H}$ : A global view of the biochemical pathways involved in the regulation of the metabolism of cancer cells. Biochim Biophys Acta 1826: 423-433, 2012.

8. Saunier E, Benelli C and Bortoli S: The pyruvate dehydrogenase complex in cancer: An old metabolic gatekeeper regulated by new pathways and pharmacological agents. Int $\mathrm{J}$ Cancer 138 : 809-817, 2016

9. Bowker-Kinley MM, Davis WI, Wu P, Harris RA and Popov KM: Evidence for existence of tissue-specific regulation of the mammalian pyruvate dehydrogenase complex. Biochem J 329 191-196, 1998

10. Mi H, Poudel S, Muruganujan A, Casagrande JT and Thomas PD: PANTHER version 10: Expanded protein families and functions, and analysis tools. Nucleic Acids Res 44: D336-D342, 2016.
11. Dupont WD and Plummer WD Jr: Power and sample size calculations. A review and computer program. Control Clin Trials 11: 116-128, 1990.

12. Ma H, Lu Y, Marchbanks PA, Folger SG, Strom BL, McDonald JA, Simon MS, Weiss LK, Malone KE, Burkman RT, et al: Quantitative measures of estrogen receptor expression in relation to breast cancer-specific mortality risk among white women and black women. Br Cancer Res 15: R90, 2013.

13. Tan LD, Xu YY, Yu Y, Li XQ, Chen Y and Feng YM: Serum HER2 level measured by dot blot: A valid and inexpensive assay for monitoring breast cancer progression. PLoS One 6: e18764, 2011.

14. Vander Heiden MG, Cantley LC and Thompson CB: Understanding the Warburg effect: The metabolic requirements of cell proliferation. Science 324: 1029-1033, 2009.

15. Jeoung NH: Pyruvate dehydrogenase kinases: Therapeutic targets for diabetes and cancers. Diabetes Metab J 39: 188-197, 2015.

16. Hur H, Xuan Y, Kim YB, Lee G, Shim W, Yun J, Ham IH and Han SU: Expression of pyruvate dehydrogenase kinase-1 in gastric cancer as a potential therapeutic target. Int J Oncol 42: 44-54, 2013.

17. Lim HY, Yip YM, Chiong E, Tiong HY, Halliwell B, Esuvaranathan $\mathrm{K}$ and Wong KP: Metabolic signatures of renal cell carcinoma. Biochem Biophys Res Commun 460: 938-943, 2015.

18. Lu CW, Lin SC, Chien CW, Lin SC, Lee CT, Lin BW, Lee JC and Tsai SJ: Overexpression of pyruvate dehydrogenase kinase 3 increases drug resistance and early recurrence in colon cancer. Am J Pathol 179: 1405-1414, 2011.

19. Roh JL, Park JY, Kim EH, Jang HJ and Kwon M: Activation of mitochondrial oxidation by PDK2 inhibition reverses cisplatin resistance in head and neck cancer. Cancer Lett 371: 20-29, 2016.

20. Gudi R, Bowker-Kinley MM, Kedishvili NY, Zhao Y and Popov KM: Diversity of the pyruvate dehydrogenase kinase gene family in humans. J Biol Chem 270: 28989-28994, 1995.

21. Lu CW, Lin SC, Chen KF, Lai YY and Tsai SJ: Induction of pyruvate dehydrogenase kinase-3 by hypoxia-inducible factor-1 promotes metabolic switch and drug resistance. J Biol Chem 283: 28106-28114, 2008.

22. Petersen TN, Brunak S, von Heijne G and Nielsen H: SignalP 4.0: Discriminating signal peptides from transmembrane regions. Nat Methods 8: 785-786, 2011.

23. Sombattheera S, Proungvitaya T, Limpaiboon T, Wongkham S, Wongkham C, Luvira V and Proungvitaya S: Total serum bile acid as a potential marker for the diagnosis of cholangiocarcinoma without jaundice. Asian Pac J Cancer Prev 16: 1367-1370, 2015.

24. Janan M, Proungvitaya S, Limpaiboon T, Proungvitaya T, Roytrakul S, Wongkham C, Jearanaikoon P, Chur-in S and Wongkham S: Serum adhesion molecule-1 (ICAM-1) as a potential prognostic marker for cholangiocarcinoma patients. Asian Pac J Cancer Prev 13 (Suppl): S107-S114, 2012. 